\title{
The correlation between longitudinal changes in hypothalamic-pituitary-adrenal (HPA)-axis activity and changes in neurocognitive function in mixed-state bipolar II disorder
}

This article was published in the following Dove Press journal:

Neuropsychiatric Disease and Treatment

Hsuan-Han Lee,' Cheng-Ho Chang,' Liang-Jen Wang, ${ }^{2}$ Chih-Ching Wu, ${ }^{3,4} \mathrm{Hsing}$ Ling Chen, ${ }^{5} \mathrm{Ti} \mathrm{Lu},{ }^{\prime} \mathrm{Ru}$-Band Lu $^{6,7}$ Sheng-Yu Lee ${ }^{1,6,8,9}$

'Department of Psychiatry, Kaohsiung Veterans General Hospital,

Kaohsiung, Taiwan; ${ }^{2}$ Department of Child and Adolescent Psychiatry, Kaohsiung Chang Gung Memorial Hospital and Chang Gung University College of Medicine, Kaohsiung, Taiwan; ${ }^{3}$ Department of Medical Biotechnology and Laboratory Science, College of Medicine, Chang Gung University, Taoyuan, Taiwan; ${ }^{4}$ Department of OtolaryngologyHead \& Neck Surgery, Chang Gung Memorial Hospital, Linkou, Taiwan; ${ }^{5}$ Department of Nuclear Medicine, Kaohsiung Veterans General Hospital, Kaohsiung, Taiwan; 'Department of Psychiatry, National Cheng Kung University Hospital, Tainan, Taiwan; ${ }^{7}$ Institute of Behavioral Medicine Sciences, College of Medicine and Hospital, National Cheng Kung University, Tainan, Taiwan; ${ }^{8}$ Department of Psychiatry, Faculty of Medicine, Kaohsiung Medical University, Kaohsiung, Taiwan; 'Department of Psychiatry, College of Medicine, National Yang-Ming University, Taipei, Taiwan

Correspondence: Sheng-Yu Lee Department of Psychiatry, Kaohsiung Veterans General Hospital, 386 Da-Chung Ist Road, Kaohsiung, Taiwan Tel +88673422121

Email shirleylee.ncku@gmail.com
Introduction: In this study, we aim to determine 1) the differences in cortisol in patients with bipolar II disorder (BD-II) and control subjects and 2) the correlation between cortisol levels and cognitive function in patients with BD-II during a 24-week follow-up period.

Methods: We recruited a total of $32 \mathrm{BD}-\mathrm{II}$ patients and 30 healthy control subjects. The BD-II patients were assessed for clinical severity and serum cortisol level at baseline and at weeks 8 , 16, and 24. The Brief Assessment of Cognition in Affective Disorders (BACA) was adopted to evaluate cognitive function at baseline and endpoint (week 24). Meanwhile, we assessed the controls for serum cortisol level and BACA at baseline.

Results: We observed that the BD-II group had a higher serum cortisol level and lower BACA composite scores compared with the healthy controls at baseline. A significant correlation was found between changes in Verbal Fluency, a subset of BACA, and changes in serum cortisol level after the 24-week follow-up, controlling for age, gender, years of education, and clinical severity $(P<0.001)$.

Conclusion: We propose that serum cortisol may be involved in the psychopathological mechanisms of cognitive decline in BD-II.

Keywords: bipolar II disorder, cognitive function, cortisol level, correlation

\section{Introduction}

A subtype of bipolar disorder (BD), bipolar II disorder (BD-II), is chronic with frequent and intense depressive episodes. ${ }^{1}$ Patients with BD-II often receive delayed diagnoses and miss out on timely treatment because they frequently have positive experiences during hypomanic episodes. ${ }^{2}$ Previous evidence has indicated that patients with BD-II are at a greater risk of both attempting and committing suicide than patients with bipolar I disorder (BD-I) or major depressive disorder due to its prolonged course, and hence the condition results in a greater burden both on patients and society.,4

Various studies have observed cognitive impairment across several domains in BD including executive functions, processing speed, attention, memory, and social cognition during acute and euthymic states. ${ }^{5-7}$ Previous studies have shown that poorer cognitive function was more common in BD-II than in BD-I. ${ }^{8}$ Cognitive impairment may subsequently have a negative effect on drug adherence and recovery of BD-II. ${ }^{8}$ Glahn et $\mathrm{al}^{9}$ reported that BD patients in the remission stage continued to experience attention deficit, which may represent a trait rather than a state variable. ${ }^{10}$ 
Some researchers have proposed that the underlying pathophysiology of BD may be associated with cognitive decline in patients with BD. ${ }^{11,12}$

Dysregulation of the Hypothalamic-Pituitary-Adrenal (HPA) axis has been suggested to play a role not only in the pathophysiology of $\mathrm{BD},{ }^{13}$ but also in the cognitive dysfunction of BD. ${ }^{14}$ Changes in cortisol, which is the main product of the HPA axis, are considered disturbances in the HPA axis and have been associated with mood disturbances in BD. ${ }^{15}$ Both hypercortisolism and hypocortisolism were observed as HPA-axis disturbances in BD and depression, ${ }^{16,17}$ and were associated with a lower quality of life. Relative hypocortisolism was found to be accompanied by a heightened level of inflammation, ${ }^{18}$ which is considered the pathogenesis of depression. ${ }^{19}$ A meta-analysis reported greater morning cortisol levels in BD patients in the euthymic state compared with controls. ${ }^{15}$ Furthermore, a lower chronic cortisol concentration may predict longer periods of stability in affective disorders. ${ }^{20}$ Since cortisol exerts fundamental homeostatic effects on cognitive and affective processes, changes in cortisol levels have been found to affect cognitive function. ${ }^{21}$ In both animal and human models, cortisol demonstrated a reversible negative effect on cognition. ${ }^{22}$ While a significant correlation between depression and cortisol level has been reported, ${ }^{23}$ recent studies have found no association between either the awakening cortisol level ${ }^{24}$ or the chronic cortisol concentration ${ }^{20}$ with cognitive impairment in any domain. However, few studies have focused on the correlation between the alteration of cortisol and cognitive function in BD-II patients.

Both cortisol level and cognitive function change depending on the state of the disease. However, previous studies have mainly focused on cross-sectional associations instead of the longitudinal association of cognitive function and cortisol. We believe that a longitudinal approach may better reflect the correlation between changes in cortisol, clinical symptoms, and cognitive function from a withinindividual perspective. Therefore, the purposes of this study were to investigate 1) the differences in cortisol level and cognitive function between BD-II patients and controls at baseline and 2) the correlation between longitudinal changes of cortisol level and cognitive function after a 24-week follow-up period.

\section{Methods}

The Institutional Review Board for the Protection of Human Subjects at Kaohsiung Veteran's General Hospital has examined and approved this research protocol. Prior to the start of the study, we completely described the study to all participants and obtained their written informed consent.

\section{Patient selection}

We recruited BD-II patients between 18 and 65 years old from acute ward and outpatient clinics of the Psychiatric Department at Kaohsiung Veterans General Hospital. All patients were first diagnosed by a senior psychiatrist according to the criteria of the Diagnostic and Statistical Manual of Mental Disorders, fourth edition (DSM-IV-TR). ${ }^{25}$ Then, the patients were given a structured interview by a clinical psychologist using the Chinese Version of the Modified Schedule of Affective Disorder and Schizophrenia-Life Time, ${ }^{26}$ which has good inter-rater reliability, ${ }^{27}$ in order to confirm the DSM-IV-TR diagnosis. Since recent studies have suggested that a 2-day duration of hypomania is more prevalent in the community than a 4-day duration of hypomania, ${ }^{28-33}$ we adopted the 2-day minimum for hypomania when diagnosing BD-II in the current study. Patients with any major mental illnesses besides BD-II, such as borderline personality disorder, substance-use disorder, and cognitive disorders, were excluded, as were patients taking over-the-counter medications, such as statins, that may have affected their metabolic profile.

None of the recruited patients had a previous history of taking mood stabilizers or antipsychotics. We assessed symptom severity at baseline and performed regular follow-up evaluations each month for next 6 months. The patients were evaluated using clinical assessments of mood symptoms and the collection of blood samples at baseline, as well as at the end of every 2-month period (points of assessment: baseline, week 8, week 16, and week 24). We used the 17-item Hamilton Depression Rating Scale (HDRS) ${ }^{34}$ to evaluate the severity of depressive symptoms and the 11-item Young Mania Rating Scale (YMRS) to determine the severity of manic symptoms. ${ }^{35}$ Throughout the follow-up period, all patients received open-label valproate treatment (500 $\mathrm{mg}$ and $1,000 \mathrm{mg}$ daily [50-100 $\mu \mathrm{g} / \mathrm{mL}$ in plasma]). We also allowed up to $8 \mathrm{mg}$ of lorazepam per day for nighttime sedation or agitation during the study. Other permitted concomitant medication included fluoxetine ( $\leq 20 \mathrm{mg} /$ daily) for depressive symptoms and risperidone ( $\leq 3 \mathrm{mg} /$ daily) for associated psychotic symptoms.

Twenty milliliters of whole blood were drawn from the antecubital vein of each patient to analyze serum cortisol concentrations at baseline and at weeks 8, 16, and 24. Before the blood sampling, we instructed the patients to avoid stress and unusual physical activity for a period of 24 hours. 
Between 8:00 and 10:00 am, after the patients had fasted for 8 hours and rested for 20 minutes, we collected fasting serum samples of hormones. The Brief Assessment of Cognition in Affective Disorders (BACA), a neuropsychological test, was performed upon recruitment, as well as at the end of the follow-up period at 24 weeks, right after the patient's blood was drawn.

\section{Healthy controls}

Thirty healthy volunteers were recruited from both the community and the hospital, with the majority coming from the health check-up clinic in the Family Medicine Department. We excluded individuals from the control group if they had any major or minor mental illness (mood disorders, anxiety disorder, psychotic disorder, personality disorder, substanceuse disorders) or a family history of psychiatric disorder among their first-degree relatives.

Following the initial evaluation, the healthy subjects had their blood drawn and their serum cortisol levels analyzed as baseline levels. They also received a one-time neuropsychological evaluation at baseline using BACA.

\section{Neuropsychological testing}

We adopted the BACA to evaluate objective cognitive functioning in patients with mood disorders. ${ }^{36}$ This instrument consists of seven subtests, including Verbal Memory (List Learning), Working Memory (Digit Sequencing), Processing Speed (Verbal Fluency; Token Motor Task; Symbol Coding), Reasoning and Problem Solving (Tower of London [TOL]), and tests of affective interference (emotional distractibility and affective memory) and emotional disinhibition, which are then summed up as affective composite scores. ${ }^{36,37}$ This assessment takes approximately 45 minutes.

The score for each BACA subtest was standardized using $\mathrm{T}$ - or z-scores. The composite z-score of BACA was the summation of the $z$-score of each BACA subtest determined by comparing a patient's performance in each subtest to that of the control group. ${ }^{36,38-40}$ A T-score of 50 demonstrates average functioning with regard to the healthy population with the same age and gender; the SD was 10 points. In the current study, we utilized the T-score developed from the Mandarin-speaking norm, ${ }^{37}$ which was already controlled for age and gender for statistical analysis.

\section{Blood assays}

Serum cortisol levels were measured with radioimmunoassay using commercially available kits (IBL Gesellschaft
Für Immunchemie Und Immunbiologie MBH, Hamburg, Germany).

\section{Statistical methods}

We utilized the statistical software package SPSS (Version $18.0)$ to analyze the data results, and $P$-values less than 0.05 were considered statistically significant.

We adopted independent $t$-tests to analyze the numerical data related to the differences in clinical characteristics and cognitive performance at baseline between BD-II patients and healthy controls and the chi-squared test to analyze differences in categorical demographic variables. The correlations between baseline cortisol level and baseline cognitive function in both BD-II patients and controls were analyzed using the Pearson correlation.

We then used multiple linear regression models to evaluate 1) the change of clinical characteristics over the 24-week follow-up period and 2) the possible correlations of cortisol levels with BACA performance. The generalized estimating equation (GEE) approach was developed to analyze longitudinal or repeatedly measured research designs in order to produce more efficient and unbiased regression estimates. ${ }^{41}$ The GEE uses the generalized linear model to estimate more efficient and unbiased regression parameters compared with ordinary least squares regression models in part because GEE allows for the specifications of a working correlation matrix that accounts for the form of the within-subject correlation of responses on dependent variables of such different distributions as normal, binomial, and Poisson. The GEE models use all the data available for each subject and can handle missing data in longitudinal studies under the assumption that such data are missing completely at random. ${ }^{42}$ Therefore, the parameter estimates may be compromised if the probability of any missing data depends on the previous values of the dependent variable. ${ }^{43}$ In order to assess the changes in clinical characteristics during the 24-week follow-up, we set the cortisol level and BACA with each subscore as independent variables. The treatment duration was also set as an independent variable, which was considered the effect of change over time. Since the GEE models use all the data available for each subject, we used all the cortisol samples available from each assessment for analysis. To evaluate the correlations between change of cortisol levels and BACA performance, we set each outcome (BACA with each subscore) as a dependent variable. In each model, cortisol, treatment duration, years of education, and HDRS and YMRS scores were included as independent variables. We interpreted the effect of cortisol 
as the relationship between the change of cortisol and the change of cognitive performance.

\section{Results}

\section{Participants characteristics}

We recruited a total of $32 \mathrm{BD}-\mathrm{II}$ patients and 30 healthy control subjects. The baseline characteristics of these participants are shown in Table 1. The categories of affective states were based on clinical evaluation according to the HDRS and YMRS rating scales without applying duration criteria: depressive (HDRS-17>8 and YMRS $<8$ ), hypomanic (YMRS $>7$ and HDRS-17<8), and mixed state (HDRS-17>7 and YMRS > 7). ${ }^{44}$ Compared with the healthy controls, the BD-II group was noted to be significantly older with fewer years of education, higher serum cortisol levels, and poorer performance in the BACA (composite scores). The study flow is shown in Figure 1.

Table 2 provides the details of patients' characteristics during the entire follow-up period. Only a total of 10 patients completed the entire 24-week follow-up period. We observed that both HDRS and YMRS improved significantly after 24 weeks of treatment $(P<0.001)$. The changes in cortisol level and cognitive function of each subtest of BACA are shown in Figures 2 and 3. A significant improvement was also noted in Verbal Fluency and several subtests in affective interference and emotional disinhibition (Table 2). However, the significance in improvement of Verbal Fluency did not survive correction for multiple comparison by setting $P<0.05 / 8=0.00625$ as significant. Moreover, we found no significant changes in the cortisol level or performance of the BACA composite score and other subscores after completing the follow-up.

Finally, we evaluated the correlation between change of cortisol levels and change in BACA performance following the 24-week follow-up period. We found a significant correlation between changes in Verbal Memory $(P<0.001)$, Token Motor $(P=0.003)$, and Verbal Fluency $(P<0.001)$, as subsets of BACA, and changes of serum cortisol level after controlling for clinical severity (HDRS and YMRS scores) and years of education. The significant correlation between changes in Token Motor $(P=0.003)$, Verbal Fluency $(P<0.001)$, and Tower of London $(P=0.002)$ with changes of serum cortisol level remains even after controlling for clinical severity (HDRS and YMRS scores), years of education, medication, type of mood episode, borderline features, past psychotic episode, and other Axis I disorders (Table 3). However, neither the changes in the BACA composite score nor other subsets correlated with changes in serum cortisol

Table I Comparison of baseline characteristics of the BD-Il patients and healthy controls

\begin{tabular}{|c|c|c|c|c|}
\hline & BD-II & $\begin{array}{l}\text { Healthy } \\
\text { controls }\end{array}$ & $\mathbf{T}$ & $P$-value \\
\hline Number, $\mathrm{n}$ & 32 & 30 & & \\
\hline Gender, n (male/female) & $12 / 22$ & $8 / 22$ & & \\
\hline Age, years (mean $\pm S D)$ & $37.9 \pm 10.8$ & $30.4 \pm 5.8$ & 3.5 & 0.001 \\
\hline Years of education, years (mean $\pm S D$ ) & $13.9 \pm 2.2$ & $16.4 \pm 1.3$ & 5.2 & $<0.001$ \\
\hline Age at onset, years (mean $\pm S D$ ) & $13.8 \pm 4$ & & & \\
\hline HDRS score (mean $\pm S D)$ & $15.5 \pm 3.6$ & & & \\
\hline YMRS score (mean \pm SD) & $11.7 \pm 3.1$ & & & \\
\hline Cortisol, $\mu g / d L ~(m e a n ~ \pm S D)$ & $10.3 \pm 3.5$ & $7.4 \pm 2.3$ & 3.7 & 0.001 \\
\hline BACA (composite T score) (mean \pm SD) & $35.9 \pm 11.4$ & $50.1 \pm 12.0$ & 4.4 & $<0.001$ \\
\hline Past hospitalization, n (yes/no) & $(2 / 30)$ & & & \\
\hline Number of hospitalization for those ever hospitalized & I & & & \\
\hline Lifetime history of psychotic symptoms (yes/no) & $(0 / 32)$ & & & \\
\hline Family history of affective disorders, $\mathrm{n}$ (yes/no) & $(0 / 32)$ & & & \\
\hline \multicolumn{5}{|l|}{ Axis I comorbidity, $\mathrm{n}$} \\
\hline Panic disorder (yes/no) & ||$/ 2 \mid$ & & & \\
\hline GAD (yes/no) & $15 / 17$ & & & \\
\hline Other anxiety disorders (phobia, PTSD; yes/no) & 9/23 & & & \\
\hline Mood state at baseline (depressive/hypomanic/mixed) & $(5 / 2 / 25)$ & & & \\
\hline \multicolumn{5}{|l|}{ Medication, $\mathrm{n}$} \\
\hline Use of fluoxetine (yes/no) & $28 / 4$ & & & \\
\hline Use of valproate & $32 / 0$ & & & \\
\hline Use of risperdal & 10 & & & \\
\hline Borderline features & 7 & & & \\
\hline
\end{tabular}

Abbreviations: HDRS, Hamilton Depression Rating Scale; YMRS, Young Mania Rating Scale; BACA, Brief Assessment of Cognition in Affective Disorders; GAD, generalized anxiety disorder; PTSD, post-traumatic stress disorder; BD-II, bipolar-II disorder. 


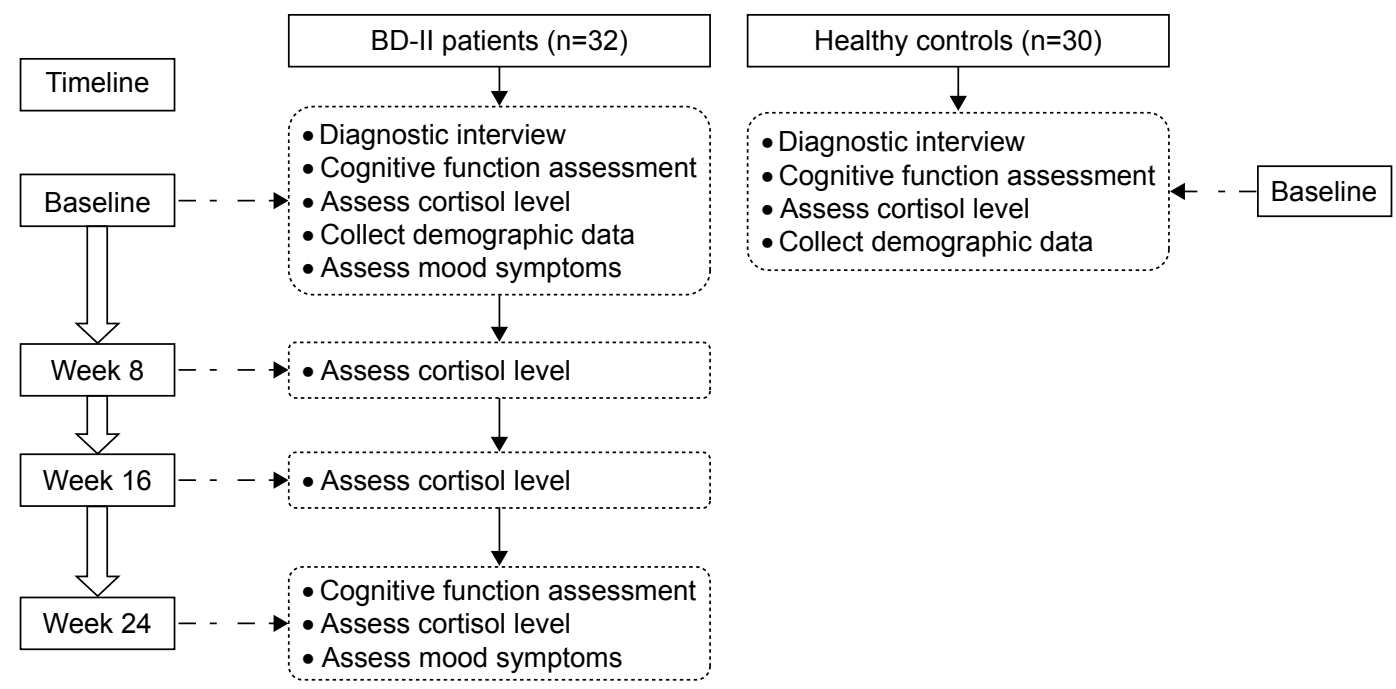

Figure I Study flow chart.

Abbreviation: BD-II, bipolar-II disorder.

Table 2 Characteristics, cortisol level, and performance of cognitive test in the BD-II patients at baseline and each follow-up

\begin{tabular}{|c|c|c|c|c|c|c|}
\hline & Baseline & Week 8 & Week 16 & Week 24 & $P$-value & $\begin{array}{l}\text { Effect size } \\
\text { (Cohen's d) }\end{array}$ \\
\hline Number, $\mathrm{n}$ & 32 & 20 & 13 & 10 & & \\
\hline Gender, n (male/female) & $12 / 22$ & $5 / 15$ & $3 / 10$ & $2 / 8$ & & \\
\hline Age, years (mean $\pm S D)$ & $37.9 \pm 10.8$ & $36.5 \pm 9.9$ & $34.2 \pm 10.1$ & $34.8 \pm 10.6$ & & \\
\hline Years of education, years (mean $\pm S D$ ) & $13.9 \pm 2.2$ & $13.4 \pm 2.2$ & $13.6 \pm 2.4$ & $14.0 \pm 2.5$ & & \\
\hline Age at onset, years (mean $\pm S D)$ & $13.8 \pm 4$ & $13.6 \pm 4.2$ & $14.0 \pm 4.8$ & $14.0 \pm 5.0$ & & \\
\hline HDRS score $($ mean $\pm S D)$ & $15.5 \pm 3.6$ & $9.7 \pm 3.2$ & $9.0 \pm 2.7$ & $9.9 \pm 1.5$ & $<0.00 I^{* *}$ & 1.43 \\
\hline YMRS score $($ mean \pm SD) & $11.7 \pm 3.1$ & $9.4 \pm 2.1$ & $8.9 \pm 2.7$ & $8.4 \pm 2.0$ & $<0.00 I^{* *}$ & 1.06 \\
\hline Mood state at baseline, $\mathrm{n}$ (depressive/hypomanic/mixed) & $(5 / 2 / 25)$ & & & $(3 / 0 / 7)$ & & \\
\hline Cortisol, $\mu \mathrm{g} / \mathrm{dL}($ mean $\pm \mathrm{SD})$ & $10.3 \pm 3.5$ & II.I \pm 4.9 & $10.5 \pm 3.7$ & $9.4 \pm 4.5$ & 0.67 & 0.12 \\
\hline \multicolumn{7}{|l|}{$\mathrm{BACA}($ mean $\pm \mathrm{SD})$} \\
\hline Verbal Memory (T score) & $43.7 \pm 10.0$ & & & $44.4 \pm 10.5$ & 0.42 & 0.15 \\
\hline Token Motor (T score) & $45.9 \pm 8.7$ & & & $46.4 \pm 5.9$ & 0.34 & 0.18 \\
\hline Digit Sequencing (T score) & $44.2 \pm 8.1$ & & & $46.0 \pm 4.0$ & 0.43 & 0.15 \\
\hline Verbal Fluency (T score) & $39.9 \pm 10.8$ & & & $41.8 \pm 11.0$ & $0.008 * *$ & 0.51 \\
\hline Symbol Coding (T score) & $38.3 \pm 10.6$ & & & $36.0 \pm 13.1$ & 0.69 & 0.08 \\
\hline Tower of London (T score) & $47.2 \pm 12.2$ & & & $48.6 \pm 12.7$ & 0.48 & 0.14 \\
\hline BACA composite scores & $31.9 \pm 19.0$ & & & $33.9 \pm 20.7$ & 0.07 & 0.35 \\
\hline \multicolumn{7}{|l|}{ Affective interference test (mean $\pm S D$ ) } \\
\hline Total affective scores & $44.1 \pm 6.7$ & & & $42.5 \pm 9.1$ & 0.38 & 0.17 \\
\hline Total non-affective scores & $47.1 \pm 10.1$ & & & $39.9 \pm 5.8$ & $<0.00 I^{* *}$ & 0.72 \\
\hline Cued affective words & $45.5 \pm 6.4$ & & & $43.6 \pm 5.9$ & 0.29 & 0.23 \\
\hline Cued non-affective words & $51.8 \pm 6.5$ & & & $43.1 \pm 7.0$ & $0.001 * *$ & 0.67 \\
\hline \multicolumn{7}{|l|}{ Affective interference test: delayed (mean $\pm S D$ ) } \\
\hline Correct affective words & $54.2 \pm 4.2$ & & & $51.0 \pm 7.7$ & 0.21 & 0.24 \\
\hline Non-affective correct words & $52.1 \pm 4.7$ & & & $47.0 \pm 9.3$ & 0.06 & 0.36 \\
\hline Affective false alarms & $46.4 \pm 10.4$ & & & $47.1 \pm 8.5$ & 0.75 & 0.06 \\
\hline Non-affective false alarms & $46.3 \pm 7.7$ & & & $46.6 \pm 7.9$ & 0.87 & 0.03 \\
\hline \multicolumn{7}{|l|}{ Emotion inhibition test (mean $\pm S D$ ) } \\
\hline Color naming score & $40.7 \pm 7.7$ & & & $41.2 \pm 5.0$ & 0.97 & 0.01 \\
\hline Neutral color word score & $39.4 \pm 8.0$ & & & $38.6 \pm 7.3$ & 0.45 & 0.15 \\
\hline Affective color word score & $38.5 \pm 9.2$ & & & $38.9 \pm 7.4$ & 0.015 & 0.47 \\
\hline Neutral word score & $38.6 \pm 10.1$ & & & $41.6 \pm 7.0$ & 0.36 & 0.18 \\
\hline Affective composite T score & $39.1 \pm 9.3$ & & & $34.9 \pm 8.4$ & 0.13 & 0.29 \\
\hline BACA (composite T score) & $35.9 \pm 11.4$ & & & $33.1 \pm 11.4$ & 0.53 & 0.12 \\
\hline
\end{tabular}

Notes: We adopted the GEE method to evaluate the change of clinical characteristics over the 24-week follow-up period by setting each BACA subscore as an independent variable and the treatment duration as an independent variable as well, which was interpreted as the effect of change over time. $* * P<0.05$.

Abbreviations: HDRS, Hamilton Depression Rating Scale; YMRS, Young Mania Rating Scale; BACA, Brief Assessment of Cognition in Affective Disorders; GEE, generalized estimating equation; BD-II, bipolar-II disorder. 


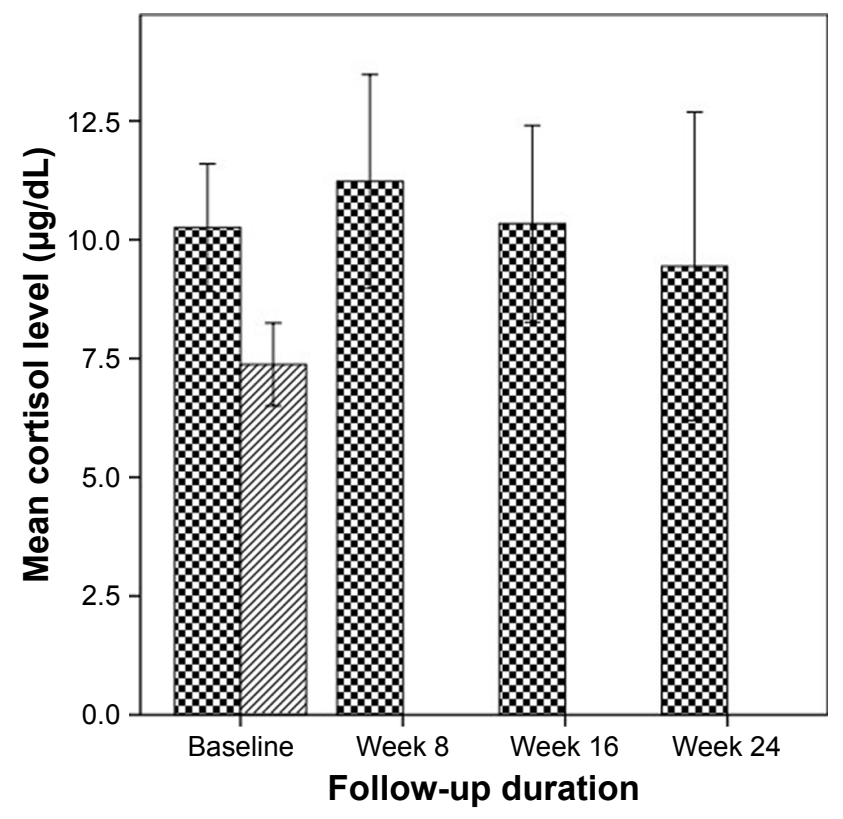

BD-II $\square$ Normal controls

Figure 2 Changes in cortisol level at baseline and endpoint. Abbreviation: BD-II, bipolar-II disorder.

level (Table 3). We observed no significance in the correlations between baseline cortisol level and baseline cognitive function in either BD-II patients or healthy control subjects (Table S1). The correlation between levels of cortisol and cognitive testing (BACA) only in mixed-state BD-II patients $(\mathrm{N}=25)$ over 24 weeks of follow-up is shown in Table S2.

\section{Discussion}

Our study reported higher serum cortisol levels and lower BACA composite scores at baseline in BD-II patients compared with the healthy controls. We also found a significant correlation between longitudinal changes in serum cortisol level and longitudinal changes in Verbal Fluency, a BACA subset, after the 24-week follow-up period, controlling for age, gender, clinical severity, and years of education.

Our results finding a higher serum cortisol level in BD-II patients compared with healthy controls agreed with a previous study of BD-I patients. ${ }^{15}$ Since all of our BD-II patients had been previously diagnosed, they had never been exposed to lithium in the past. Our patients had both elevated HDRS and YMRS scores, indicating irritability. Therefore, our results also agree with Valiengo et al,${ }^{45}$ who demonstrated that elevated cortisol levels were positively associated with irritability (dysphoria).

In the current study, we observed significant improvement in both Verbal Fluency and clinical severity in the BD-II group after 24 weeks of treatment. However, no significant improvement was noticed in BACA composite scores or BACA subscores. Since we found a significant correlation between changes in Verbal Fluency and serum cortisol level after the 24-week follow-up, Verbal Fluency may possibly be highly affected by manic symptoms and related to disease state. Our results partially agree with those of Solé et al, ${ }^{46}$ who reported that half of BD-II patients were cognitively impaired even during the euthymic state. In a 6-week follow-up study, $\mathrm{Xu}$ et $\mathrm{a}^{47}$ also observed cognitive impairment in executive function and memory. Our study tried to prolong the follow-up and treatment period to 24 weeks, but we only found significant improvement in one aspect of cognitive function, while the others remained unimproved. Our finding may indicate that cognitive dysfunction is a trait but not a state for BD-II. Furthermore, we reported no significant decrease in cortisol level after 24 weeks of treatment. Since no previous studies have investigated the longitudinal change of cortisol in BD-II over a 24-week span, our study provides initial evidence that greater morning cortisol levels may also be a trait of BD-II.

We initially found no significant correlations between baseline cortisol level and baseline cognitive function in either BD-II patients or healthy controls. Our cross-sectional finding agrees with the study of van der Werf-Eldering et al, ${ }^{24}$ who reported no association between cortisol level and cognitive function in patients with BD. Furthermore, we found no significant correlation between longitudinal change of BACA composite score and cortisol level changes in BD-II after 24 weeks of follow-up. A 5-year longitudinal study also reported no correlation between changes in cortisol and cognitive performance in a community-dwelling population. ${ }^{48}$ Herane-Vives et $\mathrm{al}^{20}$ also reported no association between chronic cortisol concentration and cognitive impairment in patients with BD-I. However, no such longitudinal study has yet been performed for BD-II. In the BACA subsets, we found a significant correlation between changes in Token Motor Task and Verbal Fluency and serum cortisol level after the 24-week follow-up period, after controlling for clinical severity, years of education, disease state, medication used, and borderline features. Our finding partially agrees with a previous 4-year prospective study ${ }^{49}$ that found a significant association between change in cortisol level and Verbal Fluency, but only in elderly women in community dwellings. The Verbal Fluency tasks in BACA are divided into two parts: in the first part, participants are given 1 minute each to come up with words in the animal category starting with a provided mandarin word, thus involving several cognitive activities, including working memory, cognitive flexibility, and self-monitoring ${ }^{50}$ which are mediated by both 
A

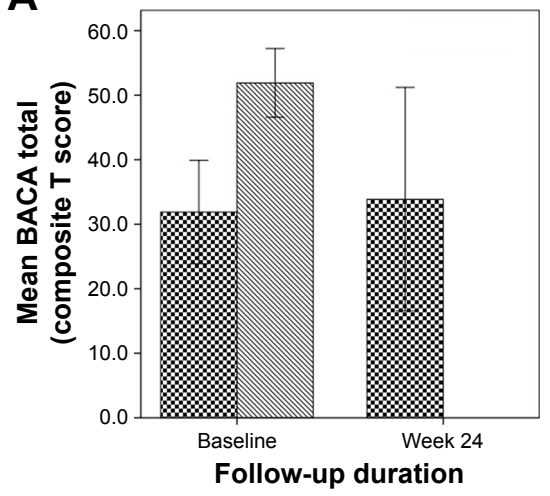

D

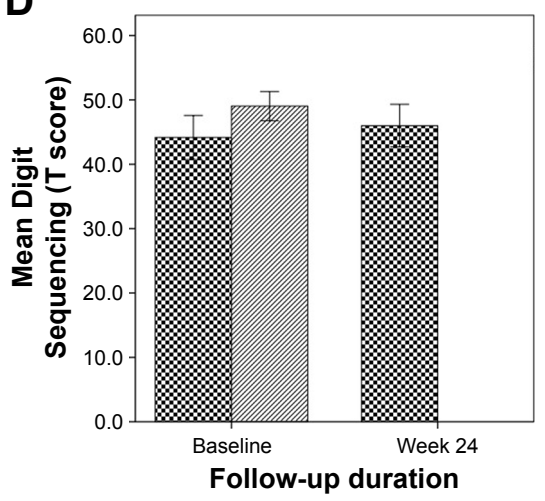

G
B

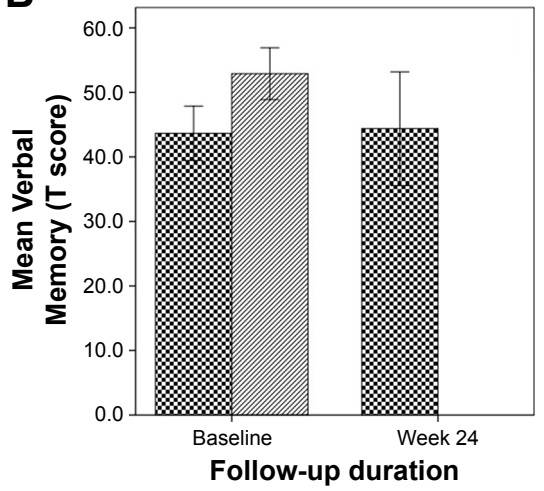

E

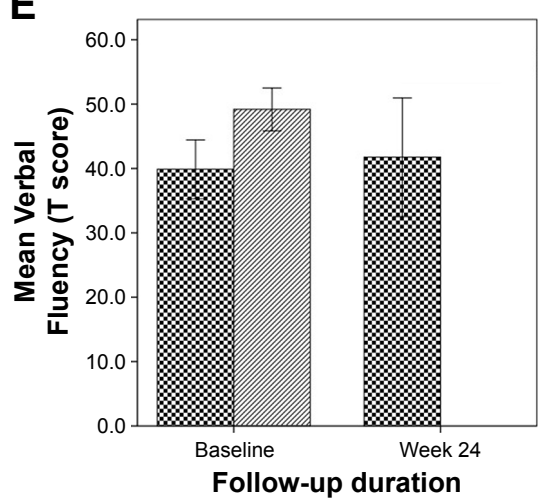

C

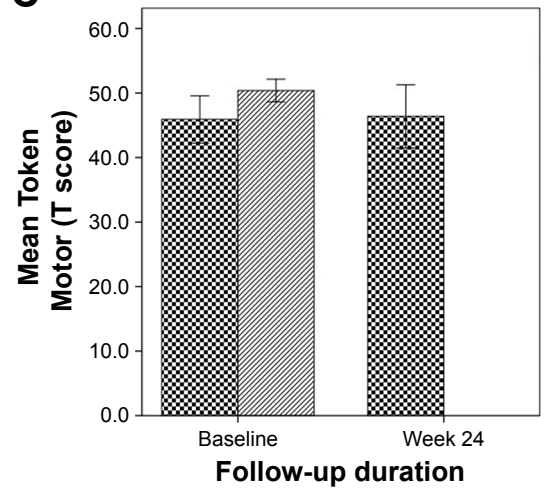

$\mathbf{F}$

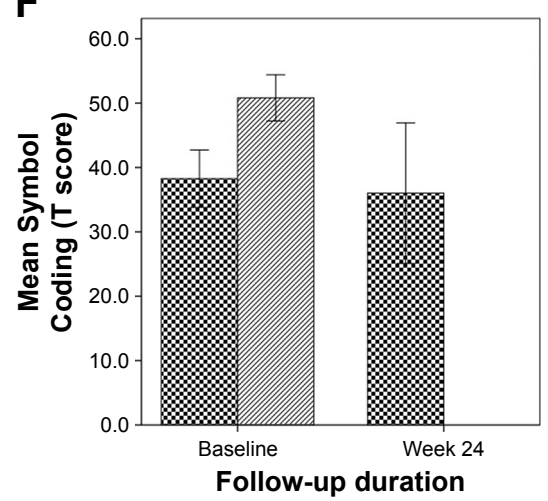

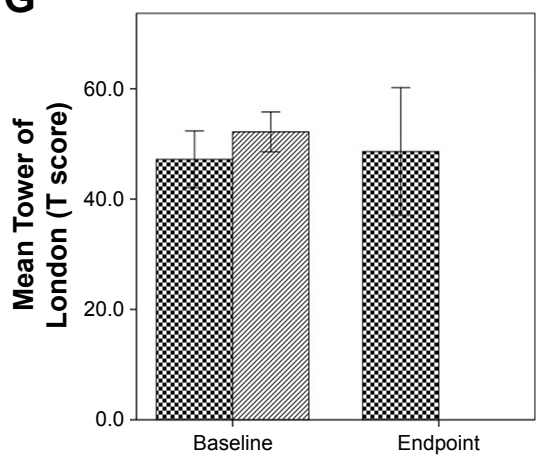

Follow-up duration
H

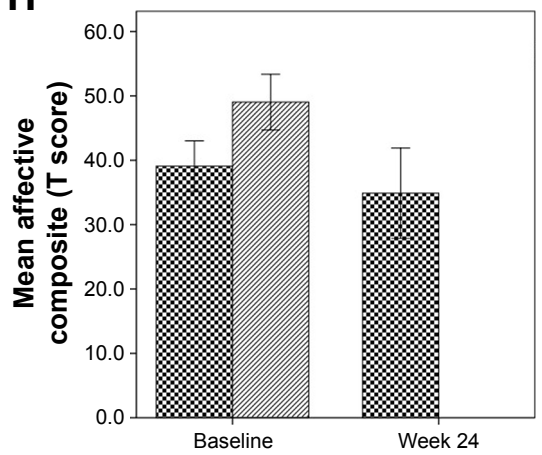

Follow-up duration

B BD-II $\square$ Normal controls

Figure 3 Changes of BACA and all subscores of BACA at baseline and endpoint (week 24).

Notes: (A) Mean BACA total (composite T score); (B) Mean Verbal Memory (T score); (C) Mean Token Motor (T score); (D) Mean Digit Sequencing (T score); (E) Mean Verbal Fluency (T score); (F) Mean Symbol Coding (T score); (G) Mean Tower of London (T score); (H) Mean affective composite (T score).

Abbreviations: BACA, Brief Assessment of Cognition in Affective Disorders; BD-II, bipolar-II disorder.

the temporal and frontal cortex. ${ }^{51} 11$ beta-Hydroxysteroid dehydrogenase type 1 (11beta-HSD1) catalyzes the change of circulating inert cortisone to cortisol. The mRNA for 11 betaHSD1 is expressed in the human hippocampus and frontal cortex. In aging humans, administration of the inhibitor for 11 beta-HSD1 was shown to improve Verbal Fluency. ${ }^{52}$ Our finding further supports the correlation between cortisol level and Verbal Fluency. Nevertheless, a specific mechanistic study is still necessary to clarify such correlations. Token
Motor Task represents the motor function of the patients. Our finding also agrees with a past finding that serum cortisol concentration correlated negatively to motor speed, psychomotor speed, and reaction time in postmenopausal women..$^{53}$ On the other hand, the inflammatory change observed during cortisol disturbances ${ }^{18}$ may also influence cognitive function, and an inverse relationship between C-reactive protein and cognitive function was reported in $\mathrm{BD} .{ }^{54}$ Previous studies have associated cognitive function decline with an increase 
Table 3 Correlation between levels of cortisol and cognitive testing (BACA) over 24 weeks of follow-up

\begin{tabular}{|c|c|c|c|c|c|c|}
\hline & \multicolumn{3}{|c|}{ Cortisol } & \multicolumn{3}{|c|}{ Cortisol } \\
\hline & \multicolumn{3}{|c|}{ Model A } & \multicolumn{3}{|c|}{ Model B } \\
\hline & B & Wald $\chi^{2}$ & $P$-value & B & Wald $\chi^{2}$ & $P$-value \\
\hline BACA total (composite T score) & 0.38 & 0.2 & 0.65 & -0.78 & 0.19 & 0.66 \\
\hline Verbal Memory (T score) & -3.9 & 15.7 & $<0.001 *$ & 1.28 & 11.85 & $0.001 *$ \\
\hline Token Motor (T score) & 1.4 & 8.6 & $0.003 *$ & 1.36 & 8.60 & $0.003 *$ \\
\hline Digit Sequencing (T score) & 0.26 & 0.85 & 0.36 & 0.20 & 0.27 & 0.60 \\
\hline Verbal Fluency (T score) & -2.2 & 88.1 & $<0.001 *$ & -2.24 & 89.86 & $<0.00 I^{*}$ \\
\hline Symbol Coding (T score) & 0.02 & $<0.001$ & 0.98 & 0.15 & 0.16 & 0.69 \\
\hline Tower of London (T score) & 0.93 & 0.24 & 0.62 & 2.16 & 9.49 & $0.002 *$ \\
\hline Affective composite (T score) & 0.57 & 1.29 & 0.26 & 0.36 & 1.35 & 0.25 \\
\hline
\end{tabular}

Notes: Controlling for age, gender, years of education, and scores of HDRS and YMRS. We adopted the GEE method to evaluate the correlations between change of cortisol levels and changes in performance of BACA by setting each outcome (BACA with each subscore) as a dependent variable. Model A: controlled for treatment duration, years of education, and HDRS and YMRS scores. Model B: controlled for treatment duration, years of education, HDRS and YMRS scores, type of drug treatment (fluoxetine yes/ no; risperidone yes/no), type of mood episode (depressive, hypomanic, mixed), presence of borderline PD features, past hospitalization, lifetime of psychotic symptoms, family history of affective disorder, and axis I comorbidity. $* P<0.05 / 8=0.00625$ as significance.

Abbreviations: BACA, Brief Assessment of Cognition in Affective Disorders; HDRS, Hamilton Depression Rating Scale; PD, personality disorder; YMRS, Young Mania Rating Scale; GEE, generalized estimating equation.

in inflammatory markers in healthy subjects. ${ }^{55}$ However, we did not include cytokine levels in our assessment. Additional studies of the correlation between change of cytokine and cortisol are warranted. Furthermore, we did not find any correlation of changes in cortisol levels with other subsets of BACA including Symbol Coding, TOL, or Affective scores. Since Verbal Fluency tasks require several cognitive processes, as previously mentioned, ${ }^{50}$ Verbal Fluency may be more vulnerable to the organic influence of hypercortisolism.

Our study has some limitations that should be mentioned. First, after 24 weeks of follow-up, our study number $(\mathrm{N}=10)$ is relatively small and thus has limited power. Since the current study is a pilot study, increasing the sample size and decreasing the drop-out rate in a future study are needed to confirm our current findings. If we had only used the most conservative method of analyzing only the 10 patients left at week 24 and the same 10 patients at baseline and eliminated the other 22 patients who left the study early, none of the correlations between change of cortisol level and change of cognitive function would be significant. Therefore, the current study results need to be interpreted with caution. In addition, the 24-week duration was designed to avoid learning effect. A cognitive test which could be repeated more frequently with little learning effect may be needed to decrease the follow-up duration and the high dropout rate. The present study also did not evaluate or control for stressful life events, which may also be highly correlated with cortisol levels. When evaluating cognitive function, it is necessary to consider the influence of mood symptoms. Even in healthy controls, subthreshold mood symptoms may affect some neurocognitive dysfunctions. However, in the current study, we did not evaluate the HDRS and YMRS scores in the normal controls. We will try to include this assessment in future studies. In addition, euthymic BD-II patients were not included in this study either. However, we controlled for mood symptom when evaluating the correlation between cortisol and cognitive function in BD-II patients. Third, we used the norm developed for BACA scores for the Mandarin-speaking population ${ }^{37}$ instead of the English norms. ${ }^{36}$ Again, our study results should be interpreted with caution since they may differ for the English norms. ${ }^{36}$ Furthermore, the control sample differs significantly from the bipolar sample with regard to age and years of education. Since cortisol levels may increase with age ${ }^{56}$ and cognitive function decline may be related to the years of education, ${ }^{57} \mathrm{a}$ future study would need a control sample that more closely matches the patient group. We also adopted the 2-day duration for diagnosing hypomania instead of the 4-day duration provided in the DSM-IV-TR criteria. The current positive findings may not be generalizable to studies that adopt the DSM-IV-TR criteria for hypomania. Moreover, cortisol levels vary throughout the day. Therefore, the 2-hour window (between 8 and $10 \mathrm{am}$ ) of collection time may be too wide, which may negatively affect the current findings. However, we had to provide the convenience of arriving between 8 and 10 am for the patients and controls since blood samples were collected in the hospital. Future studies with a more confined window of collection time may be required. Since the use of antidepressants may contribute to the regulation of the cortisol system, ${ }^{58}$ the change of cortisol level in the current study may differ from the BD patients who did not receive antidepressants in their first episode. Nevertheless, we attempted to control the use of antidepressants and other medications in all the analyses to minimize such influence. In addition, some demographic data were still lacking which 
should be added in future studies: the number of past mood episodes including hypomanic, depressive, mixed episodes; whether past episodes were seasonal pattern or rapid cycling, the patient's marital status; and axis I comorbidity (other than borderline personality disorder). Finally, the longitudinal correlation between other neuroendocrine parameters and cognitive function with even longer follow-up periods are necessary to support the current findings.

In conclusion, we observed increased serum cortisol levels and poorer cognitive performance in patients with BD-II compared to healthy controls. We also report a significant correlation between longitudinal changes in serum cortisol level and cognitive function after 24 weeks of standard treatment. Based on our findings, we propose that serum cortisol may be involved in the psychopathological mechanisms of cognitive decline in BD-II patients. Although studies with larger populations are needed in the future, our results can serve as a reference for treatments that decrease cortisol, as a new therapeutic alternative, for attenuating cognitive decline in BD-II patients.

\section{Acknowledgments}

The authors would like to thank Ms Yu-Hsuan Lin and Ms Yi-Chin Wang for their assistance in preparing this manuscript.

This work was supported in part by MOST 103-2622B-006-006-CC2 (to R-BL), MOST 103-2314-B-075B006 (to S-YL) from the Taiwan Ministry of Science and Technology; VGHKS104-098 (to S-YL), VGHKS105-122 (to S-YL), and VGHKS107-153 (to S-YL) from Kaohsiung Veterans General Hospital, Taiwan.

\section{Author contributions}

H-HL wrote the first draft, S-YL gave conceptual advice and revised the manuscript. S-YL, C-CW, H-LC, and L-JW managed the lab work and statistics. C-HC and TL managed participant recruitment. R-BL supervised this work and edited the manuscript. All authors contributed toward data analysis, drafting and critically revising the paper and agree to be accountable for all aspects of the work.

\section{Disclosure}

The authors report no conflicts of interest in this work.

\section{References}

1. Judd LL, Akiskal HS. Depressive episodes and symptoms dominate the longitudinal course of bipolar disorder. Curr Psychiatry Rep. 2003;5(6):417-418.

2. Angst J. The bipolar spectrum. Br J Psychiatry. 2007;190:189-191.
3. Macqueen GM, Marriott M, Begin H, Robb J, Joffe RT, Young LT. Subsyndromal symptoms assessed in longitudinal, prospective follow-up of a cohort of patients with bipolar disorder. Bipolar Disord. 2003; 5(5):349-355.

4. Rihmer Z, Szádóczky E, Füredi J, Kiss K, Papp Z. Anxiety disorders comorbidity in bipolar I, bipolar II and unipolar major depression: results from a population-based study in Hungary. J Affect Disord. 2001; 67(1-3):175-179.

5. Bora E, Hıdıroğlu C, Özerdem A, et al. Executive dysfunction and cognitive subgroups in a large sample of euthymic patients with bipolar disorder. Eur Neuropsychopharmacol. 2016;26(8):1338-1347.

6. Bourne C, Aydemir Ö, Balanzá-Martínez V, et al. Neuropsychological testing of cognitive impairment in euthymic bipolar disorder: an individual patient data meta-analysis. Acta Psychiatr Scand. 2013;128(3): 149-162.

7. Cardenas SA, Kassem L, Brotman MA, Leibenluft E, Mcmahon FJ. Neurocognitive functioning in euthymic patients with bipolar disorder and unaffected relatives: A review of the literature. Neurosci Biobehav Rev. 2016;69:193-215.

8. Summers M, Papadopoulou K, Bruno S, Cipolotti L, Ron MA. Bipolar I and bipolar II disorder: cognition and emotion processing. Psychol Med. 2006;36(12):1799-1809.

9. Glahn DC, Bearden CE, Niendam TA, Escamilla MA. The feasibility of neuropsychological endophenotypes in the search for genes associated with bipolar affective disorder. Bipolar Disord. 2004;6(3): 171-182.

10. Quraishi S, Frangou S. Neuropsychology of bipolar disorder: a review. J Affect Disord. 2002;72(3):209-226.

11. Bauer IE, Pascoe MC, Wollenhaupt-Aguiar B, Kapczinski F, Soares JC. Inflammatory mediators of cognitive impairment in bipolar disorder. J Psychiatr Res. 2014;56:18-27.

12. Zhao L, Lin Y, Lao G, et al. Association study of dopamine receptor genes polymorphism with cognitive functions in bipolar I disorder patients. J Affect Disord. 2015;170:85-90.

13. Belvederi Murri M, Prestia D, Mondelli V, et al. The HPA axis in bipolar disorder: Systematic review and meta-analysis. Psychoneuroendocrinology. 2016;63:327-342.

14. Daban C, Vieta E, Mackin P, Young AH. Hypothalamic-pituitaryadrenal axis and bipolar disorder. Psychiatr Clin North Am. 2005; 28(2):469-480.

15. Girshkin L, Matheson SL, Shepherd AM, Green MJ. Morning cortisol levels in schizophrenia and bipolar disorder: a meta-analysis. Psychoneuroendocrinology. 2014;49:187-206.

16. Bremmer MA, Deeg DJ, Beekman AT, Penninx BW, Lips P, Hoogendijk WJ. Major depression in late life is associated with both hypo- and hypercortisolemia. Biol Psychiatry. 2007;62(5):479-486.

17. Maripuu M, Wikgren M, Karling P, Adolfsson R, Norrback KF. Relative hypo- and hypercortisolism are both associated with depression and lower quality of life in bipolar disorder: a cross-sectional study. PLoS One. 2014;9(6):e98682.

18. Wikgren M, Maripuu M, Karlsson T, et al. Short telomeres in depression and the general population are associated with a hypocortisolemic state. Biol Psychiatry. 2012;71(4):294-300.

19. Dowlati Y, Herrmann N, Swardfager W, et al. A meta-analysis of cytokines in major depression. Biol Psychiatry. 2010;67(5):446-457.

20. Herane-Vives A, Cleare AJ, Chang CK, et al. Cortisol levels in fingernails, neurocognitive performance and clinical variables in euthymic bipolar I disorder. World J Biol Psychiatry. 2017:1-12.

21. Heffelfinger AK, Newcomer JW. Glucocorticoid effects on memory function over the human life span. Dev Psychopathol. 2001;13(3): 491-513.

22. Lupien SJ, Mcewen BS. The acute effects of corticosteroids on cognition: integration of animal and human model studies. Brain Res Brain Res Rev. 1997;24(1):1-27.

23. Reppermund S, Zihl J, Lucae S, et al. Persistent cognitive impairment in depression: the role of psychopathology and altered hypothalamicpituitary-adrenocortical (HPA) system regulation. Biol Psychiatry. 2007;62(5):400-406. 
24. van der Werf-Eldering MJ, Riemersma-van der Lek RF, Burger H, Holthausen EA, Aleman A, Nolen WA. Can variation in hypothalamicpituitary-adrenal (HPA)-axis activity explain the relationship between depression and cognition in bipolar patients? PLoS One. 2012; 7(5):e37119.

25. American Psychiatric Association. Diagnostic and Statistical Manual of Mental Disorder-IV-TR. 4th ed. Washington: American Psychiatric Association; 2000.

26. Endicott J, Spitzer RL. A diagnostic interview: the schedule for affective disorders and schizophrenia. Arch Gen Psychiatry. 1978; 35(7):837-844.

27. Huang SY, Lin WW, Ko HC, Hc K, et al. Possible interaction of alcohol dehydrogenase and aldehyde dehydrogenase genes with the dopamine D2 receptor gene in anxiety-depressive alcohol dependence. Alcohol Clin Exp Res. 2004;28(3):374-384.

28. Akiskal HS, Djenderedjian AM, Rosenthal RH, Khani MK. Cyclothymic disorder: validating criteria for inclusion in the bipolar affective group. Am J Psychiatry. 1977;134(11):1227-1233.

29. Akiskal HS, Rosenthal RH, Rosenthal TL, Kashgarian M, Khani MK, Puzantian VR. Differentiation of primary affective illness from situational, symptomatic, and secondary depressions. Arch Gen Psychiatry. 1979;36(6):635-643.

30. Angst J. The emerging epidemiology of hypomania and bipolar II disorder. J Affect Disord. 1998;50(2-3):143-151.

31. Angst J, Gamma A, Sellaro R, Lavori PW, Zhang H. Recurrence of bipolar disorders and major depression. A life-long perspective. Eur Arch Psychiatry Clin Neurosci. 2003;253(5):236-240.

32. Benazzi F. Is 4 days the minimum duration of hypomania in bipolar II disorder? Eur Arch Psychiatry Clin Neurosci. 2001;251(1):32-34.

33. Judd LL, Akiskal HS, Schettler PJ, et al. The comparative clinical phenotype and long term longitudinal episode course of bipolar I and II: a clinical spectrum or distinct disorders? J Affect Disord. 2003; 73(1-2):19-32.

34. Hamilton M. Development of a rating scale for primary depressive illness. Br J Soc Clin Psychol. 1967;6(4):278-296.

35. Young RC, Biggs JT, Ziegler VE, Meyer DA. A rating scale for mania: reliability, validity and sensitivity. Br J Psychiatry. 1978;133: 429-435.

36. Keefe RS, Fox KH, Davis VG. The Brief Assessment of Cognition. (BAC-A): performance of patients with bipolar depression and healthy controls. Journal of affective disorders Disorders Affective, editor; 2014; 166:86-860.

37. Lee CY, Lee SY, Huang YC, et al. The Chinese version of the Brief Assessment of Cognition in Affective Disorders: normative data of a Mandarin-speaking population. Clin Neuropsychol. 2017:1-14.

38. Wang LJ, Lin PY, Lee Y, et al. Validation of the Chinese version of Brief Assessment of Cognition in Schizophrenia. Neuropsychiatr Dis Treat. 2016;12:2819-2826.

39. Wang LJ, Huang YC, Hung CF, et al. The Chinese version of the Brief Assessment of Cognition in Schizophrenia: Data of a largescale Mandarin-speaking population. Arch Clin Neuropsychol. 2017; 32(3):289-296.

40. Lee SY, Wang LJ, Chang CH, et al. Serum DHEA-S concentration correlates with clinical symptoms and neurocognitive function in patients with bipolar II disorder: A case-controlled study. Prog Neuropsychopharmacol Biol Psychiatry. 2017;74:31-35.
41. Zeger SL, Liang KY, Albert PS. Models for longitudinal data: a generalized estimating equation approach. Biometrics. 1988;44(4): 1049-1060.

42. Shen CW, Chen YH. Model selection for generalized estimating equations accommodating dropout missingness. Biometrics. 2012;68(4): 1046-1054.

43. Liu J, Colditz GA. Optimal design of longitudinal data analysis using generalized estimating equation models. Biom J. 2017;59(2):315-330.

44. Zimmerman M, Martinez JH, Young D, Chelminski I, Dalrymple K. Severity classification on the Hamilton Depression Rating Scale. J Affect Disord. 2013;150(2):384-388.

45. Valiengo LL, Soeiro-de-Souza MG, Marques AH, et al. Plasma cortisol in first episode drug-naïve mania: differential levels in euphoric versus irritable mood. J Affect Disord. 2012;138(1-2):149-152.

46. Solé B, Jiménez E, Torrent $\mathrm{C}$, et al. Cognitive variability in bipolar II disorder: who is cognitively impaired and who is preserved. Bipolar Disord. 2016;18(3):288-299.

47. Xu G, Lin K, Rao D, et al. Neuropsychological performance in bipolar I, bipolar II and unipolar depression patients: a longitudinal, naturalistic study. J Affect Disord. 2012;136(3):328-339.

48. Singh-Manoux A, Dugravot A, Elbaz A, Shipley M, Kivimaki M, Kumari M. No evidence of a longitudinal association between diurnal cortisol patterns and cognition. Neurobiol Aging. 2014;35(10): 2239-2245.

49. Beluche I, Carrière I, Ritchie K, Ancelin ML. A prospective study of diurnal cortisol and cognitive function in community-dwelling elderly people. Psychol Med. 2010;40(6):1039-1049.

50. Schwartz S, Baldo J, Graves RE, Brugger P. Pervasive influence of semantics in letter and category fluency: a multidimensional approach. Brain Lang. 2003;87(3):400-411.

51. Baldo JV, Schwartz S, Wilkins D, Dronkers NF. Role of frontal versus temporal cortex in verbal fluency as revealed by voxel-based lesion symptom mapping. J Int Neuropsychol Soc. 2006;12(6):896-900.

52. Sandeep TC, Yau JL, Maclullich AM, et al. 11Beta-hydroxysteroid dehydrogenase inhibition improves cognitive function in healthy elderly men and type 2 diabetics. Proc Natl Acad Sci U S A. 2004;101(17): 6734-6739.

53. Raczkiewicz D, Sarecka-Hujar B, Owoc A, Bojar I. Cognitive functions and serum cortisol concentration in perimenopausal and postmenopausal women working non-manually. Neuro Endocrinol Lett. 2017;38(4):269-274.

54. Dickerson F, Stallings C, Origoni A, Vaughan C, Khushalani S, Yolken R. Elevated C-reactive protein and cognitive deficits in individuals with bipolar disorder. J Affect Disord. 2013;150(2):456-459.

55. Goldstein FC, Zhao L, Steenland K, Levey AI. Inflammation and cognitive functioning in African Americans and Caucasians. Int J Geriatr Psychiatry. 2015;30(9):934-941.

56. Halbreich U, Asnis GM, Zumoff B, Nathan RS, Shindledecker R. Effect of age and sex on cortisol secretion in depressives and normals. Psychiatry Res. 1984;13(3):221-229.

57. Farmer ME, Kittner SJ, Rae DS, Bartko JJ, Regier DA. Education and change in cognitive function. The Epidemiologic Catchment Area Study. Ann Epidemiol. 1995;5(1):1-7.

58. Piwowarska J, Dryll K, Szelenberger W, Pachecka J. Cortisol level in men with major depressive disorder treated with fluoxetine or imipramine. Acta Pol Pharm. 2008;65(1):159-164. 


\section{Supplementary materials}

Table S I Correlations between cortisol and all BACA scales at baseline

\begin{tabular}{|c|c|c|c|c|}
\hline \multirow[t]{2}{*}{ Test and subtests } & \multicolumn{2}{|l|}{ BD-II } & \multicolumn{2}{|c|}{ Controls } \\
\hline & $\boldsymbol{r}$ & $P$-value & $r$ & $P$-value \\
\hline BACA (composite T score) & -0.11 & 0.65 & -0.14 & 0.48 \\
\hline Verbal Memory (T score) & 0.17 & 0.48 & -0.20 & 0.32 \\
\hline Digit Sequencing (T score) & -0.08 & 0.74 & 0.04 & 0.86 \\
\hline Token Motor (T score) & -0.28 & 0.26 & -0.13 & 0.53 \\
\hline Verbal Fluency (T score) & -0.20 & 0.42 & -0.001 & 0.99 \\
\hline Symbol Coding (T score) & -0.25 & 0.30 & -0.08 & 0.70 \\
\hline Tower of London (T score) & 0.13 & 0.61 & -0.14 & 0.47 \\
\hline Affective composite (T score) & -0.09 & 0.71 & -0.10 & 0.63 \\
\hline
\end{tabular}

Notes: Pearson correlation was used to evaluate the correlation between cortisol level and BACA performances in all the BD-II patients at baseline.

Abbreviation: BACA, Brief Assessment of Cognition in Affective Disorders; BD-II, bipolar II disorder.

Table S2 Correlation between levels of cortisol and cognitive testing (BACA) in mixed-state BD-II patients over 24 weeks of follow-up

\begin{tabular}{|c|c|c|c|c|c|c|}
\hline & \multicolumn{3}{|c|}{ Cortisol } & \multicolumn{3}{|c|}{ Cortisol } \\
\hline & \multicolumn{3}{|c|}{ Model A } & \multicolumn{3}{|c|}{ Model B } \\
\hline & $\bar{B}$ & Wald $\chi^{2}$ & $P$-value & $\overline{\mathbf{B}}$ & Wald $\chi^{2}$ & $P$-value \\
\hline BACA total (composite T score) & $-0.6 I$ & 0.15 & 0.70 & -0.61 & 0.75 & 0.70 \\
\hline Verbal Memory (T score) & -0.2 & 0.5 & 0.58 & $\mathrm{I} . \mathrm{I}$ & 5.2 & 0.024 \\
\hline Token Motor (T score) & -0.02 & 0.005 & 0.94 & -0.02 & 0.002 & 0.97 \\
\hline Digit Sequencing (T score) & 0.1 & 0.06 & 0.81 & 0.43 & 2.80 & 0.10 \\
\hline Verbal Fluency (T score) & -2.3 & 1,662 & $<0.001 *$ & -2.26 & 1,656 & $<0.00 I^{*}$ \\
\hline Symbol Coding (T score) & 0.28 & 0.07 & 0.79 & 0.23 & 0.32 & 0.57 \\
\hline Tower of London (T score) & -0.32 & 0.06 & 0.81 & 2.99 & 11.67 & $0.001 *$ \\
\hline Affective composite (T score) & 0.12 & 0.06 & 0.81 & 1.00 & 9.8 & $0.002 *$ \\
\hline
\end{tabular}

Notes: Controlling for age, gender, and years of education, and scores of HDRS and YMRS. $* P<0.05 / 8=0.00625$ as significance. We adopted the GEE method to evaluate the correlations between change of cortisol levels and changes in performance of BACA by setting each outcome (BACA with each subset) as a dependent variable. Model A: controlled for treatment duration, years of education, and HDRS and YMRS scores. Model B: controlled for treatment duration, years of education, HDRS and YMRS scores, type of drug treatment (fluoxetine yes/no; risperidone yes/no), type of mood episode (depressive, hypomanic, mixed), presence of borderline PD features, past hospitalization, lifetime of psychotic symptoms, family history of affective disorder, and axis I comorbidity.

Abbreviations: BACA, Brief Assessment of Cognition in Affective Disorders; BD-II, bipolar II disorder; HDRS, Hamilton Depression Rating Scale; PD, personality disorder; YMRS, Young Mania Rating Scale; GEE, generalized estimating equation.

\section{Publish your work in this journal}

Neuropsychiatric Disease and Treatment is an international, peerreviewed journal of clinical therapeutics and pharmacology focusing on concise rapid reporting of clinical or pre-clinical studies on a range of neuropsychiatric and neurological disorders. This journal is indexed on PubMed Central, the 'PsycINFO' database and CAS, and is the official journal of The International Neuropsychiatric Association (INA). The manuscript management system is completely online and includes a very quick and fair peer-review system, which is all easy to use. Visit http://www.dovepress.com/testimonials.php to read real quotes from published authors. 